\title{
Reconnection and energy release rates in a two-ribbon flare ${ }^{\star}$
}

\author{
C. H. Miklenic ${ }^{1}$, A. M. Veronig ${ }^{1}$, B. Vršnak ${ }^{2}$, and A. Hanslmeier ${ }^{1}$ \\ ${ }^{1}$ Institute of Physics, University of Graz, Universitätsplatz 5, 8010 Graz, Austria \\ 2 Hvar Observatory, Faculty of Geodesy, Kačićeva 26, 1000 Zagreb, Croatia
}

Received 2 June 2006 / Accepted 13 September 2006

\section{ABSTRACT}

\begin{abstract}
Aims. The aim of this study was to derive the local reconnection rate (coronal electric field) and the global reconnection rate (magnetic flux change rate), as well as the energy release rate (Poynting flux), in a two-ribbon flare from chromospheric/photospheric observations. Furthermore, we tested whether equal shares of positive and negative magnetic flux are involved in the flare process.

Methods. A well-observed GOES M3.9 two-ribbon flare was analyzed. The required observables (ribbon expansion velocity, newly brightened area, and magnetic field strength at the ribbon front) were extracted from the TRACE $1600 \AA$ and Kanzelhöhe H $\alpha$ image time series, and a SOHO MDI magnetogram. Furthermore, the ratio of the converted positive vs. negative magnetic flux was determined. Both RHESSI hard X-ray 20-60 keV full-disk time profiles and subregion time profiles derived from a time series of RHESSI images in the same energy range were used as independent, observable proxies for the energy release rate. The RHESSI images were also used to localize the sites where the bulk of the energy was deposited by fast electrons.

Results. We found good temporal correlations between the derived time profiles (local and global reconnection rate, Poynting flux) and observed HXR flux. The local reconnection-rate peak values ranged from $2.7 \mathrm{~V} \mathrm{~cm}^{-1}$ to $11.8 \mathrm{~V} \mathrm{~cm}^{-1}$, whereas the positive and the negative magnetic flux covered by the flare emission were equal within $5-10 \%$.

Conclusions. The results indicate that the local reconnection rate, the global reconnection rate, and the energy release rate in a simple two-ribbon flare can be derived from chromospheric/photospheric observations. Furthermore, it was confirmed that equal shares of positive and negative magnetic flux participated in the reconnection process.
\end{abstract}

Key words. Sun: flares - Sun: magnetic fields - Sun: chromosphere - Sun: corona

\section{Introduction}

Magnetic energy is the only energy reservoir with sufficient capacity to fuel solar flares. We do not know, however, how the enormous amount of flare energy (up to $10^{25} \mathrm{~J}$ ) is released on a time scale of minutes or how it is converted from magnetic energy to plasma energy (heat and kinetic energy). It is now generally accepted that the reconnection of magnetic field lines is the source of this sudden energy release. The change in the connectivity of the field lines, which takes place during the reconnection process, allows the release of magnetic excess energy that is stored in a sheared or twisted field configuration. Furthermore, magnetic reconnection occurring in the corona is the only viable mechanism that can account for many well-known flare characteristics, such as the growth of the flare loop system, the hard X-ray (HXR) footpoint emission, the cusp-shaped structures observed in soft X-rays (SXR) above the loop top HXR sources, and the expansion of the $\mathrm{H} \alpha$ and UV flare emission (ribbons) away from the neutral line, which can be explained by the most widely accepted flare model, the CSHKP model. It evolved from concepts of Carmichael (1964), Sturrock (1966), Hirayama (1974), and Kopp \& Pneuman (1976), and has been further elaborated by many authors, e.g., Priest \& Forbes (1990) and Forbes $\&$ Lin (2000).

According to this model a large number of chromospheric $\mathrm{H} \alpha / \mathrm{UV}$ kernels form the two $\mathrm{H} \alpha / \mathrm{UV}$ flare ribbons, which are located on either side of the magnetic neutral line, and therefore

$\star$ Movies are only available in electronic form at http: //www . aanda.org have opposite magnetic polarities. As the flare proceeds, the diffusion region (DR), which contains the X-type reconnection point, rises, i.e., the reconnection occurs at successively higher altitudes in the corona. As a consequence, the newly created flare loops are larger than the older ones, and their footpoints lie farther apart than the footpoints of previously created loops. For this reason the flare ribbons seem to separate from each other in a direction perpendicular to the magnetic neutral line. However, this observed motion is only an apparent one, caused by the successive brightening of different regions in the chromosphere.

This paper is structured in the following way. Section 2 gives a short theoretical overview of the magnetic reconnection rates and energy release rates and of how they can be derived from observations. Section 3 contains descriptions of the analyzed tworibbon flare and the data sets used. Section 4 describes the methods that have been applied to determine the required observables. In Sect. 5 the results of the determination of the local reconnection rate $E$, the Poynting flux $S$, the magnetic flux change rate $\dot{\varphi}$, and the ratio $R$ of converted positive versus negative flux are presented and discussed. The results are summarized in Sect. 6.

\section{Magnetic reconnection and energy release rates}

The reconnection rate is one of the most important quantities in magnetic reconnection physics. Sweet (1958) and Parker (1957) developed the first reconnection model; however, the SweetParker reconnection rate turned out to be too slow to account for the sudden energy release in solar flares. The first model and best-known example of fast reconnection is the Petschek-model (Petschek 1964). In Petschek's configuration, the DR is still 
important, since the actual process of reconnection occurs there, but in contrast to the Sweet-Parker model, it is extremely small. Therefore, the propagation time through it is shorter and the reconnection process takes place faster than in the Sweet-Parker model. Moreover, the fast reconnection mechanism causes the splitting of the current sheet into two pairs of standing slowmode shocks (switch-off shocks), which are attached to the corners of the DR. Most of the plasma does not need to flow through the DR in order to be accelerated. Instead, it is heated, compressed, deflected, and accelerated at the shocks, which thus are the main sites where magnetic energy is converted into plasma energy.

At the present time there is no established theory of the physics that determines the reconnection rate. Therefore, its derivation from observations is essential. Since until now only a few direct observations of a plasma inflow into the reconnection region have been made (Yokoyama et al. 2001; Lin et al. 2005; Narukage \& Shibata 2006), and the reconnection region itself is far too small to be observed directly, indirect methods are needed to determine the reconnection rate from observations. Forbes \& Priest (1984) and Forbes \& Lin (2000) pointed out that the local reconnection rate, i. e., the rate at which magnetic field lines are carried into the reconnection site, then break and reconnect, is directly given by the coronal electric field $E$ at the reconnection site. They derived a simple relation between the local reconnection rate and the apparent motion of the chromospheric $\mathrm{H} \alpha$ or UV flare ribbons that holds in a two-dimensional configuration with translational symmetry along the third dimension. According to this relation, it is possible to determine the local reconnection rate in two-ribbon flares as the product of two observables, namely, the apparent flare-ribbon separation speed $v$, and the photospheric magnetic field strength component $B$ perpendicular to the solar surface at the current ribbon front location

$E=v B$.

Instead of the electric field, Forbes \& Lin (2000) considered the rate of photospheric magnetic flux change $\dot{\varphi}$ in the region of newly closed field lines and pointed out that the flux change rate, or the drop in total voltage along the current sheet, is a global reconnection rate that is valid in three dimensions and that describes the rate at which the net open magnetic flux is converted to closed flux

$\dot{\varphi}=\frac{\partial}{\partial t} \int B_{n} \mathrm{~d} a$,

where $B_{n}$ is the magnetic field strength component perpendicular to the solar surface in the newly brightened area $\mathrm{d} a$ that is swept by the flare ribbons.

The magnetic flux $\varphi$ that is converted from open to closed in the course of a flare originates in equal shares from the positive and negative magnetic polarity domains. Therefore, the ratio $R$ of converted positive versus negative flux

$R=\frac{\left|\varphi_{-}\right|}{\varphi_{+}}$

must be 1 over the time period of the flare $\left(\varphi_{+}=\int \dot{\varphi}_{+} \mathrm{d} t\right.$ and $\left.\varphi_{-}=\int \dot{\varphi}_{-} \mathrm{d} t\right)$. Qiu \& Yurchyshyn (2005) determined the ratio $R$ of converted positive versus negative flux. In 11 out of the 13 events analyzed, they found flux ratios ranging between 0.5 and 2. Given the uncertainties involved in the measurements, this was regarded as a good balance between converted positive and negative flux compared to the theoretically expected value of 1 .
The released magnetic energy comes from the Poynting flux $\boldsymbol{S}=\left(\boldsymbol{E} \times \boldsymbol{B}_{\mathrm{c}}\right) / \mu$ into the reconnection region (where $\mu$ is the magnetic permeability, $\boldsymbol{E}$ the coronal electric field, and $\boldsymbol{B}_{\mathrm{c}}$ the coronal magnetic field). Taking into account (1) that this flux enters into the reconnection region from both sides, (2) that the magnetic flux is conserved $\left(v_{\mathrm{i}} B_{\mathrm{c}}=v B\right.$, where $v_{\mathrm{i}}$ is the inflow velocity into the reconnection region), and (3) that proportionality is assumed between the coronal, chromospheric, and photospheric magnetic fields ( $B_{\mathrm{c}}=a B$, where $a<1$ is an unknown proportionality factor), the Poynting flux into the reconnection region can be expressed by the same observables that are used to determine the local reconnection rate, namely,

$|S|=S=\frac{2}{\mu} E B_{\mathrm{c}}=\frac{2 a}{\mu} v B^{2}$.

Therefore, the energy release rate $\dot{W}$ can be written as the product of the absolute value of the Poynting flux into the reconnection region (current sheet) and the area $A$ of the current sheet (Isobe et al. 2002). Assuming that this area does not change significantly during the flare and is independent of the magnetic field strength, the energy release rate is proportional to the Poynting flux (Asai et al. 2004)

$\dot{W}=S \cdot A=\frac{2 a A}{\mu} v B^{2}=q \frac{1}{\mu} v B^{2} \propto \frac{1}{\mu} v B^{2}$,

where the unknown proportionality factor $q=2 a \mathrm{~A}$ depends on both the area $A$ of the current sheet and the ratio $a=B_{\mathrm{c}} / B$ of coronal to photospheric magnetic field (Asai et al. 2004 estimated $a \sim 0.2$ ).

During the impulsive phase of a flare, microwave and HXR emission are often observed with very similar looking time profiles. Since both types of radiation are generated by fast electrons that have been accelerated in the course of the sudden energy release at the reconnection site, the intensity of the emission is proportional to the number of accelerated electrons, and this number is in turn proportional to the rate at which energy is released at the reconnection site. Therefore, microwave and HXR flux act as indicators for the number of accelerated particles and are considered to be proportional to the energy release rate in a solar flare (e.g., Hudson 1991). Hence, they can be used as proxies for the energy release rate or the reconnection rate, respectively, and both the evolution of the derived reconnection and energy release rate can be compared with the observed microwave and/or HXR flux time profiles. Peaks in the temporal variation of the derived profiles $E$ (local reconnection rate), $S$ or $\dot{W}$ (Poynting flux or energy release rate, respectively), and $\dot{\varphi}$ (magnetic flux change rate) are expected to occur at the same time as peaks in the observed HXR or microwave time profiles.

Up to the present, only a few attempts have been made to determine $E, \dot{W}(S), \dot{\varphi}$, or $R$ from observations. Most of these studies used $\mathrm{H} \alpha$ observations to determine $v$, although it is also possible to use UV observations (e.g., Fletcher et al. 2004). Qiu et al. (2004) derived $E$ and $\dot{\varphi}$ in 2 two-ribbon flares and found that both $E$ and $\dot{\varphi}$ were temporally correlated with the nonthermal emission of the events. Jing et al. (2005) derived $E$ and $\dot{\varphi}$ for a sample of 13 two-ribbon flares of different GOES classes. They found a high correlation between the magnitude of the GOES $\mathrm{X}$-ray flare and the maximum value of $E$. As expected, more energetic flares had higher reconnection rate peak values.

The $\mathrm{H} \alpha$ and UV flare emission can be excited nonthermally by accelerated electrons bombarding the chromosphere, as well as thermally by heat flux from the hot flaring corona - in contrast to chromospheric HXR emission, which is solely due to precipitating electrons emitting nonthermal bremsstrahlung when 
braking in the field of the ions. It is reasonable to assume that the dominant energy-transport mechanism at the flare ribbon segments associated with HXR sources are high-energy electron beams, whereas on non-HXR segments thermal conduction fronts could be dominant. However, for both energy-transport mechanisms (fast electrons/thermal conduction), the $\mathrm{H} \alpha$ and UV ribbons trace the energy released in the corona and channeled along the loops to the chromosphere.

We note that it should also be possible to derive $v$ directly from the HXR footpoint motions. However, the HXR sources most often appear very localized on specific segments of the extended $\mathrm{H} \alpha / \mathrm{UV}$ ribbons (e.g., Hoyng et al. 1981; Sakao et al. 1992; Asai et al. 2004; Krucker et al. 2005). Consequently, tracing the HXR footpoint motion and identifying it with the reconnection velocity could be misleading. The most obvious example is a situation where a new dominant HXR source appears at a new location, resulting in an apparent jump of the source. Moreover, the HXR source can "slide" along the ribbon, if the location favorable to particle acceleration shifts along the neutral line (e.g. Vršnak et al. 1987; Grigis \& Benz 2005; Bogachev et al. 2005). Similarly, measurements of the HXR source motion are misleading when reconnection takes place in highly sheared configurations (Bogachev et al. 2005). To avoid these problems, we follow the expansion of the UV and $\mathrm{H} \alpha$ flare-ribbon fronts away from the neutral line that is evidently more directly related to the reconnection rate, and the chances for misleading situations are much smaller.

Since there is evidence that local reconnection rates and energy release rates are not uniform along the flare ribbons (Asai et al. 2002; Wang et al. 2003), it makes sense to track the ribbons selectively at locations where they cross an HXR source, i.e., at locations where the bulk of the energy in nonthermal electrons is deposited. Therefore, the highest local reconnection rates can be expected there. Asai et al. (2004) tracked the ribbon motion in a two-ribbon flare along several paths, each of them crossing a different HXR source. The authors derived $E$ and $S$ for each path and compared the temporal variation in the profiles with the observed HXR light curve. They successfully reconstructed the peaks in the nonthermal emission. However, the correlation between HXR light curve and derived reconnection rate, or energy release rate was only temporary and regional, respectively; i.e., it appeared only at the time of a certain HXR peak and along the path leading across that peak. The HXR light curve was constructed by integrating the HXR flux over the whole solar disk. Consequently, this light curve showed each HXR peak of the event, irrespective of its particular location. Therefore, it should be possible to strengthen the correlation between derived reconnection rates/energy release rates and HXR light curves by using spatially-resolved HXR light curves that were derived only from that area where HXR images show the location of a particular HXR peak.

\section{Data and observations}

We analyzed a comparatively simple two-ribbon flare (GOES-class M3.9, $\mathrm{H} \alpha$ importance $2 \mathrm{~N}$, position $\mathrm{S}_{2}{ }^{\circ}, \mathrm{E}^{\circ} 7^{\circ}$ ) in the NOAA Active Region 501, which occurred on November 18,2003 , by using the following data sets.

1. A full-disk $\mathrm{H} \alpha$ image time series (377 images, pixel size $2.2^{\prime \prime}$, cadence $\sim 11 \mathrm{~s}$ ) provided by the Kanzelhöhe Solar Observatory (KSO), Austria (Otruba \& Pötzi 2003).

2. A TRACE $1600 \AA$ image sequence $(64$ images, FOV: $383.5^{\prime \prime} \times 383.5^{\prime \prime}$ centered at $x=-188^{\prime \prime}, y=8^{\prime \prime}$,

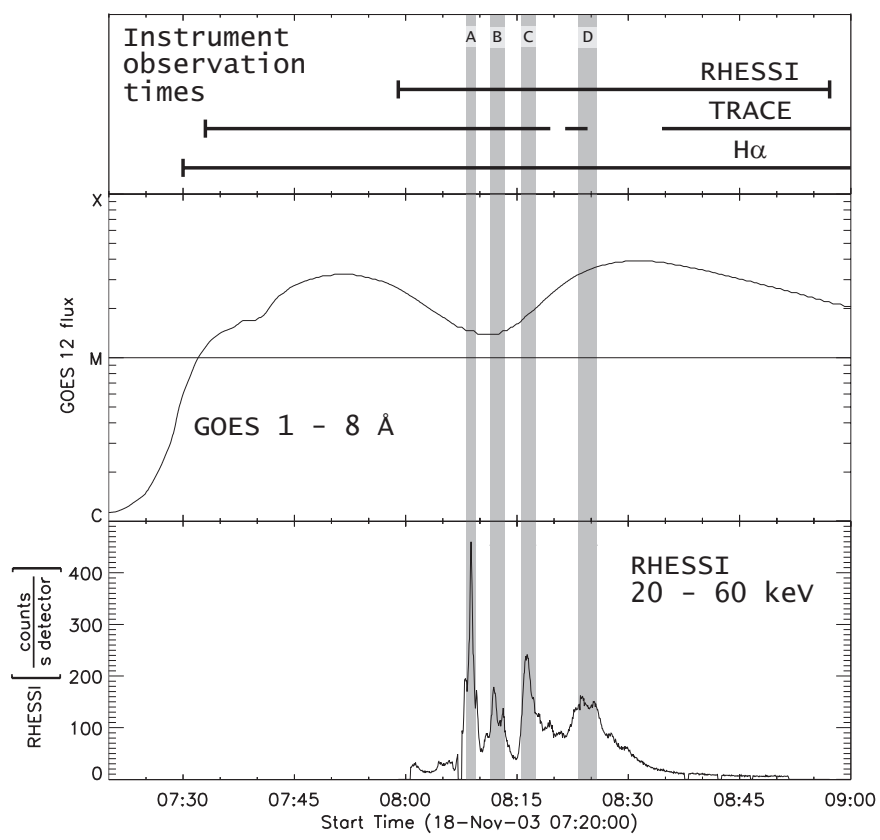

Fig. 1. Top: instrument observation time intervals (RHESSI, TRACE, KSO $\mathrm{H} \alpha$ ). Middle: GOES12 1-8 A SXR flux. Bottom: RHESSI 20-60 keV HXR time profile (counts in the course of shutter movement were set to zero). The four gray vertical bars designated as $\mathrm{A}, \mathrm{B}, \mathrm{C}$, and D highlight the main HXR bursts.

pixel size $0.5^{\prime \prime}$, cadence $\sim 23 \mathrm{~s}$ ) derived from the Transition Region and Coronal Explorer (TRACE; Handy et al. 1999).

3. A full-disk magnetogram before flare onset (06:23:02 UT, pixel size $\sim 2.0^{\prime \prime}$ ) provided by the MDI/SOI instrument (Scherrer et al. 1995) on board the Solar and Heliospheric Observatory (SOHO). The H $\alpha$, TRACE $1600 \AA$, and MDI data sets were used to determine the required observables (ribbon velocity, newly brightened area, and magnetic field strength component normal to the solar surface at the ribbon front location and within the newly brightened area, respectively).

4. A full-disk HXR intensity time profile in the energy band from 20-60 keV and an HXR image time series in the same energy band, derived from the Reuven Ramaty High Energy Solar Spectroscopic Imager (RHESSI; Lin et al. 2002). Forty-seven images (FOV: $160^{\prime \prime} \times 160^{\prime \prime}$ ) were reconstructed by means of the Clean algorithm with natural weighting using RHESSI front detector segments 3 to 8 and giving a spatial resolution of $\sim 8^{\prime \prime}$. Natural weighting was chosen, since this scheme is more sensitive to extended sources (Hurford et al. 2002, see also Veronig et al. 2006). The image integration time was $30 \mathrm{~s}$, except for 3 images where it had to be extended to $60 \mathrm{~s}$ to obtain useful images. The HXR images were used, on the one hand, to localize the sites where the bulk of the energy was deposited by fast electrons and then track the flare ribbons along paths that crossed these sites. On the other hand, individual imaging light curves were constructed from the image time series in order to reconstruct the temporal evolution of the emission in particular subareas of the flaring region. RHESSI observed 4 main HXR bursts in the course of the impulsive phase (see bottom panel of Fig. 1), designated as bursts A, B, C, and D.

To construct the imaging light curves, 4 RHESSI images were selected that showed the location of one of these bursts. Afterwards, an area in those images was chosen, which was large 

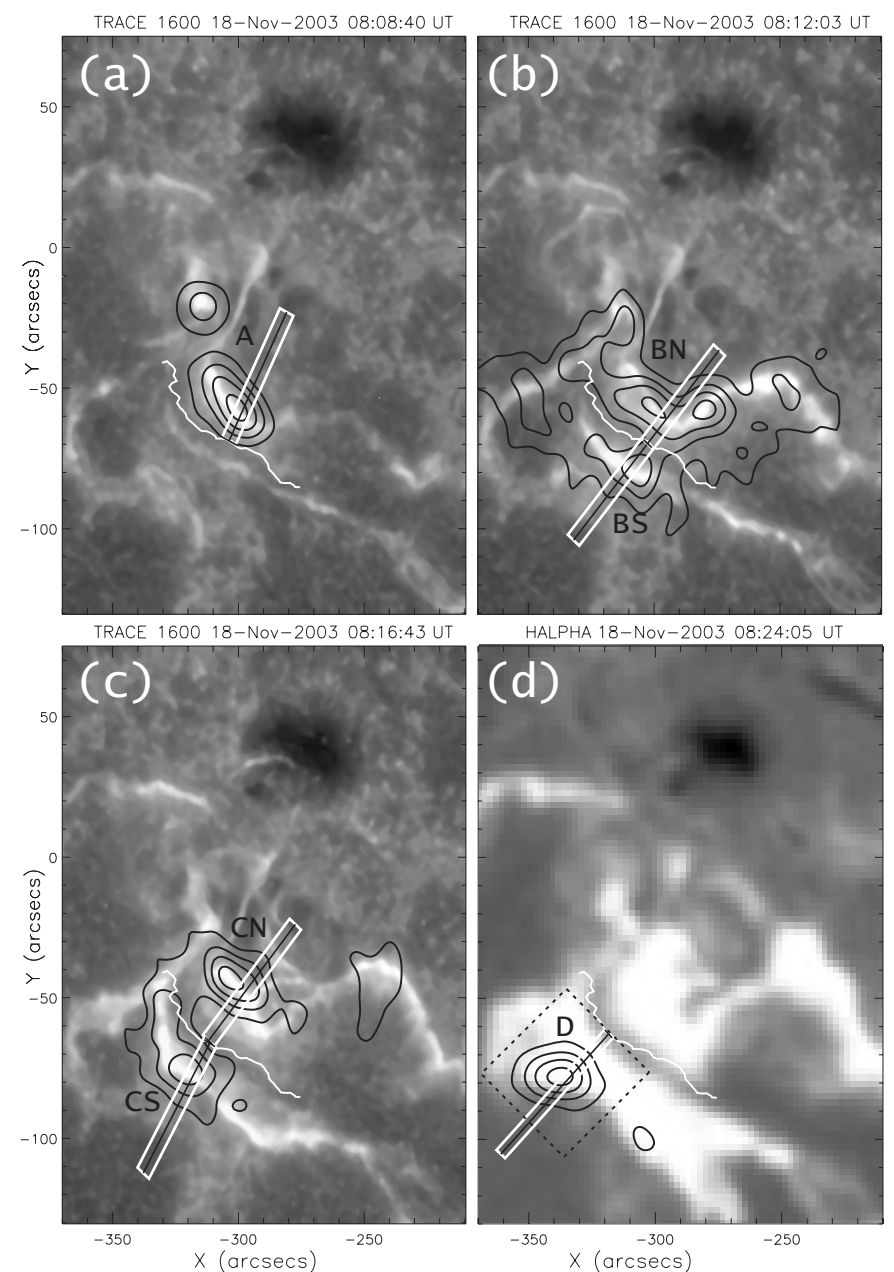

Fig. 2. HXR burst sites and location of the ribbon-tracking paths ( $\mathrm{N}$ stands for north, $\mathrm{S}$ for south of the inversion line). The HXR bursts (black contours, contour levels 30, 50, 70, 90\% of the maximum HXR 20-60 keV intensity) are situated at the outer edges of the separating flare ribbons, and indicate the sites of the actual energy deposition by accelerated electrons. White line: magnetic inversion line (contour level $0.1 \mathrm{G})$. Short black straight lines: locally-defined magnetic inversion line. Long black straight lines: direction along which the ribbon motion was tracked. White rectangles: subregions that were included in the ribbon-tracking procedure. Panels a)-c): underlying TRACE $1600 \AA$ image, d): underlying $\mathrm{H} \alpha$ image. The dashed rectangle marks the area that was used to construct the HXR burst D subregion time profile that is presented in Col. 3, row 6 of Fig. 6. - Solar north is up, west is to the right. Note that the northern ribbon evolved into a rather complex structure, whereas the southern ribbon remained in its relatively unbowed shape. FOV: $160^{\prime \prime} \times 205^{\prime \prime}$.

enough to contain the whole HXR burst along with its adjacency (Fig. 2d). Then, the maximum intensity value within this area was determined in each of the 47 images, and the smallest maximum $I_{\mathrm{s}}$ was used as a threshold; i.e., the intensities of those pixels in the subregion that exceeded $0.2 I_{\mathrm{s}}$ were added up in each image.

To accomplish the co-alignment of the different data sets, the H $\alpha$ and TRACE images were co-aligned with MDI by crosscorrelation of an MDI continuum image (06:23:23 UT) with an $\mathrm{H} \alpha$ red wing image and a TRACE WL image. In the next step, $\mathrm{H} \alpha$ line core images, as well as TRACE $1600 \AA$ images, were shifted by the resulting offset values, accounting for the different pointings of the TRACE WL and $1600 \AA$ telescopes. The
RHESSI images were visually co-aligned with the already coaligned TRACE $1600 \AA$ images as follows. After selecting pairs of HXR and TRACE images that matched best in time, the HXR contours were superimposed on the TRACE images. Because of the excellent spatial resolution of the TRACE images, the HXR contours could be easily attached to very small but bright TRACE patches.

In Fig. 1, the 4 main HXR bursts A, B, C, and D are highlighted by gray, vertical bars. Note that these bars have no relation to image integration times but act only as a visual accentuation of the peaks in the full-disk HXR 20-60 keV time profile that is presented in the bottom panel of Fig. 1. In the top panel of Fig. 1, the instrument observation time intervals are indicated for RHESSI, TRACE, and $\mathrm{H} \alpha$. The interruptions in the TRACE line mark data gaps ( $2 \mathrm{~min}$ and $11 \mathrm{~min})$ in the TRACE $1600 \AA$ image time series.

The middle panel shows the GOES12 1-8 $\AA$ SXR flux. In the time range between 07:20:00 UT and 09:00:00 UT, the GOES flux peaked two times. The first peak was GOES class M3.2, the second one M3.9. As expected, the GOES flux increased in the course of the four HXR bursts (impulsive phase) and reached its second peak value at 08:31:00 UT, i.e., shortly after the end of the fourth HXR burst. This seems to indicate the validity of the Neupert effect in this event (Neupert 1968; Hudson 1991; Dennis \& Zarro 1993; Veronig et al. 2002). RHESSI missed the first GOES SXR peak interval when the spacecraft entered the Earth shadow. Therefore, the analysis was confined to the time interval around the second GOES flux rising phase ( $\approx$ 08:00 UT-08:30 UT).

\section{Analysis}

Local reconnection rates, as well as energy release rates, were determined by using both $\mathrm{H} \alpha$ and TRACE $1600 \AA$ images. The magnetic flux change rate, however, was determined only in TRACE, since the $\mathrm{H} \alpha$ images turned out to be unsuitable in this case due to saturation effects.

\subsection{The ribbon-front tracking method}

Ribbon-tracking paths: the four main HXR bursts (Fig. 1) are located near the outer edges of the flare ribbons (see Fig. 2). Movie 1 of the online material shows the TRACE $1600 \AA$ ribbon separation with superimposed HXR contours. Since these burst locations indicate the sites where the strongest energy deposition by accelerated electrons occurs, the ribbon motion was tracked along paths that crossed a particular HXR burst site. However, since TRACE images exhibit a much higher spatial resolution than HXR images, the exact bearing of each path was selected by means of bright TRACE kernels in the regarded area. Therefore, the paths did not always cross the center of the HXR burst contours. Moreover, some images indicate HXR emission along a significant part of the elongated flare ribbons (e.g., Fig. 2b). Figure 2 shows the directions of the ribbon-tracking paths normal to the locally-defined magnetic inversion line, the subregions that were included in the ribbon-tracking procedure, and the relevant part of the magnetic inversion line (see also Fig. 3). The ribbon-tracking paths are designated as follows: A (Fig. 2a), $\mathrm{BN}$ and BS (Fig. 2b), CN and CS (Fig. 2c) - each with underlying TRACE $1600 \AA$ image, and D (Fig. 2d, with underlying H $\alpha$ image).

Figure 3 demonstrates the magnetic complexity of the flaring region and the nearby sunspots. Paths $\mathrm{CN}$ and $\mathrm{CS}$, as well as 


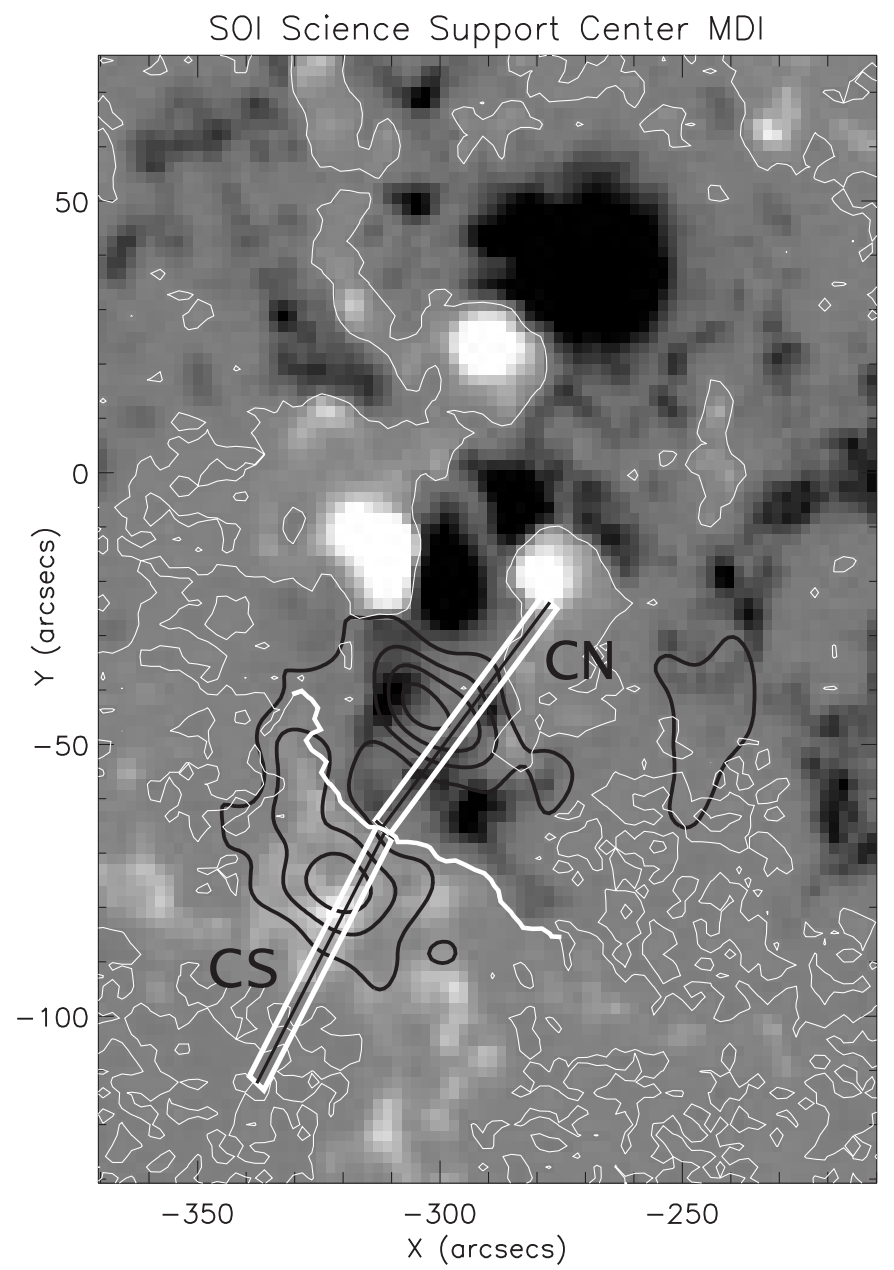

Fig. 3. HXR contours (black lines), magnetic inversion line (thin and thick white lines) and ribbon-tracking paths $\mathrm{CN}$ and CS superimposed on an MDI magnetogram (06:23:02 UT, data range scaled to $\pm 500 \mathrm{G}$ out of $\sim-1695 \mathrm{G}$ to $\sim+1216 \mathrm{G})$. Same contour levels and FOV as in Fig. 2. Black/white patches represent the negative/positive magnetic polarity domains.

the burst $\mathrm{C}$ HXR contours and the magnetic inversion line contours are superimposed on the MDI magnetogram. The northern ribbon swept the negative magnetic polarity domain (paths A, $\mathrm{BN}$, and $\mathrm{CN}$ ), whereas the southern ribbon crossed the positive domain (paths BS, CS, and D).

In Fig. 4 the HXR burst contours, along with the corresponding ribbon-tracking paths, are superimposed on the first available TRACE image. Especially north of the inversion line, the bursts are located rather close to one another, and thus the ribbontracking paths cross more than one HXR burst site.

Determination of $v$ and $B$ : the ribbon velocity was defined as the time derivative of the ribbon front distance from the locally assessed magnetic inversion line. The distances were obtained from the TRACE and $\mathrm{H} \alpha$ image time series by using intensity threshold values that were found by trial and error. The ultimate threshold values were chosen due to their suitability for identifying those pixels as ribbon pixels that would also have been chosen as ribbon pixels with the naked eye. After detecting the ribbon pixels, their distance from the locally-defined inversion line was determined. Then, all ribbon pixels with distance values $\geq 95 \%$ of the maximum distance were defined as ribbon front pixels, giving ribbon fronts of $1^{\prime \prime}-2$ " thickness. In order to smooth out small-scale inaccuracies in the image

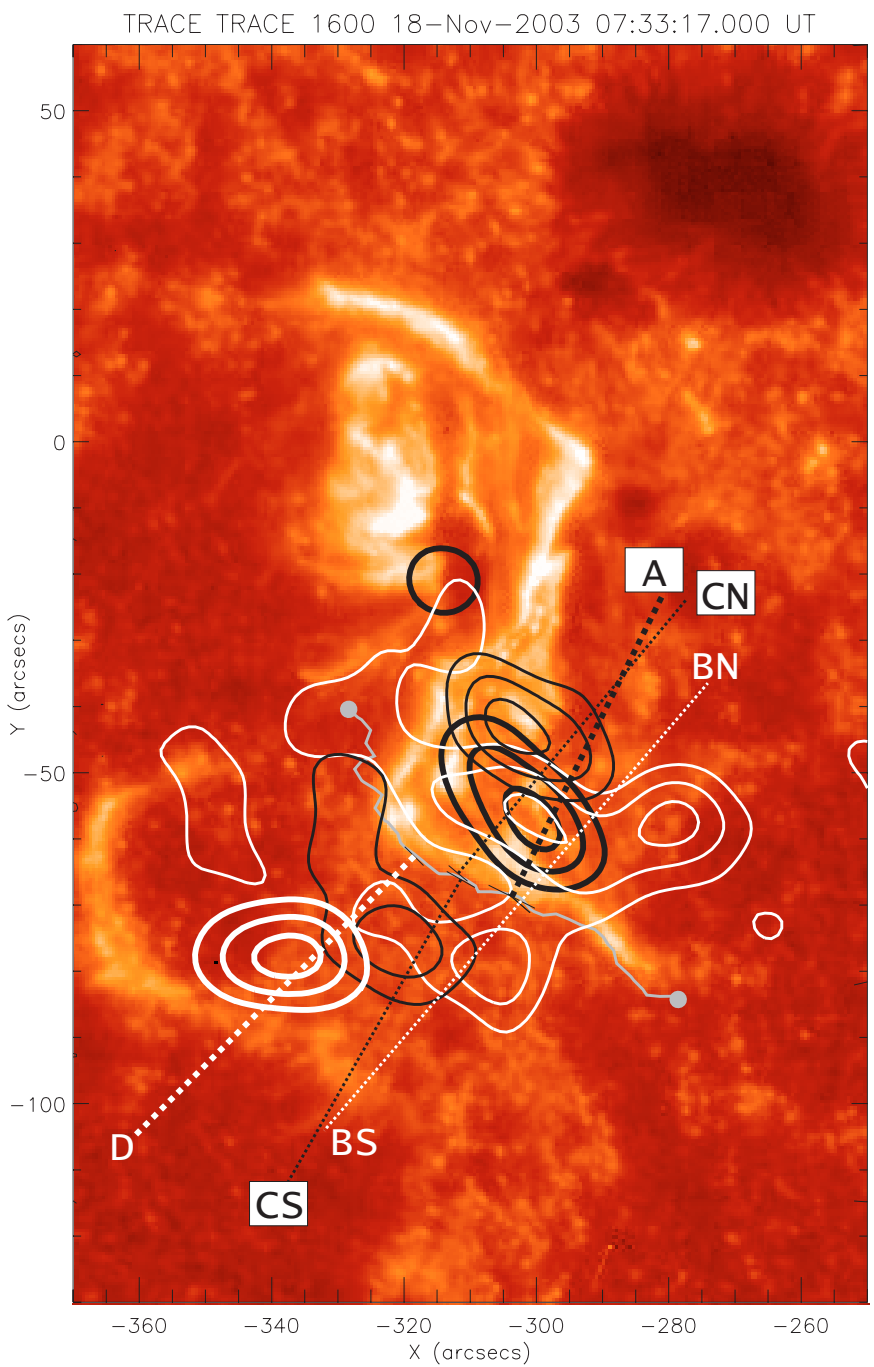

Fig. 4. HXR burst contours (solid) and ribbon-tracking paths (dotted) superimposed on a TRACE $1600 \AA$ image. Thick-black: burst A, path $\mathrm{A}$; thin-white: burst $\mathrm{B}$, paths $\mathrm{BN}$ and $\mathrm{BS}$; thin-black: burst $\mathrm{C}$, paths $\mathrm{CN}$ and $\mathrm{CS}$; and thick-white: burst D, path D. Gray line with filled dots at ends: magnetic inversion line. FOV: $120^{\prime \prime} \times 190^{\prime \prime}$.

co-alignment of the different data sets, which are unavoidable, the ultimate ribbon-front distance values, as well as the magnetic field strength values at the ribbon front, were averaged over the whole width of the analyzed subregion, i.e., the width of the white rectangles in Fig. 2, which was approximately 7".

Furthermore, a magnetic field strength adjustment was carried out, since according to Berger \& Lites (2003) the current Level-1.5 MDI full-disk calibration underestimates the flux density values. Therefore, the original magnetic field strength values were multiplied, according to Berger \& Lites (2003), by a scaling factor of 1.56, and these scaled values were used for further analysis. In order to smooth out fluctuations due to measurement uncertainties, spline smoothing was applied to both the distance and magnetic field strength data points. The splines were calculated either up to the point where the tracked part of the ribbons began to cool down, i.e., the ribbon distance did not increase anymore, or, if such a cooling was not observed within the time range of the TRACE data set, the splines were calculated up to the end of the TRACE observation time. Finally, the required temporal evolution of the apparent ribbon velocity was 
determined as the time derivative of the spline-smoothed distance profiles.

\subsection{Newly brightened area, converted magnetic flux, and magnetic flux change rate}

The newly brightened area (NBA) in an image compared to the preceding images was determined separately for each magnetic polarity domain. For that purpose, the first TRACE image (07:59:51 UT) was subtracted from each element of the image time series to eliminate bright remnants from the previous flare event, and then an intensity threshold value was used to detect flare pixels in the difference images. To find an appropriate threshold, the smallest intensity maximum $\left(I_{\mathrm{sm}}\right)$ of the entire difference image time series was determined, and then this value was multiplied by various scaling factors $(0.8-1.5)$ to get a set of potential threshold values. Out of this set, the ultimate threshold value $\left(1.1 I_{\mathrm{sm}}\right)$ was chosen due to its suitability to detect newly brightened flare pixels at the ribbon fronts not only in the brightest sections of the flare ribbons but also in fainter ones that were located a long way from the HXR burst sites, without identifying non-flare pixels erroneously as flare pixels.

In order to be counted among the newly brightened pixels, a particular pixel had to fulfill the following conditions: (1) its intensity value had to exceed the given threshold; (2) the same pixel had to be a non-flare pixel in the preceding images; (3) it had to be located inside the currently analyzed magnetic polarity domain and exceed the MDI noise level of $\pm 20 \mathrm{G}$.

After detecting the newly brightened pixels in an image, the magnetic field strength values at these pixel locations were taken. Then Eq. (2) was approximated by a sum; i.e., at each time step the magnetic field strength values of the newly brightened flare pixels in a given polarity domain were summed up do give $\dot{\varphi}_{+}$and $\dot{\varphi}_{-}$. The mean of these two profiles gave $\dot{\varphi}$. Afterwards, the converted magnetic flux for each magnetic polarity domain was determined by the time integrals of $\dot{\varphi}_{+}$and $\dot{\varphi}_{-}$to compute the flux ratio $R$ (Eq. (3)). Movie 2 of the online material shows the detection of the NBA, which is predominantly located at the ribbon fronts (red/blue area: positive/negative magnetic polarity domain).

\section{Results}

\subsection{Local reconnection rate and energy release rate}

Local reconnection rates, as well as energy release rates, were derived from both $\mathrm{H} \alpha$ and TRACE observations. The results were similar, but we present only the TRACE results due to the better quality of the TRACE data (higher spatial resolution, no seeing effects). However, in one case (path D), we present the $\mathrm{H} \alpha$ results, because the TRACE data showed gaps in the time range for HXR burst $\mathrm{D}$. $\mathrm{H} \alpha$ results for the remaining paths can be found in Miklenic (2005) ${ }^{1}$.

The electric field strength (local reconnection rate) and the Poynting flux (energy release rate) were derived in two steps: (1) calculation of the two observables (chromospheric ribbon velocity $v$ and photospheric line-of-sight magnetic field strength $B$ ) by applying the method described in Sect. 4.1, and (2) application of Eqs. (1) and (5). Note that the real Poynting flux $S=2 a v B^{2} / \mu$ is lower than the plotted quantity $v B^{2} / \mu$; $a$ is the

\footnotetext{
1 Note that in Miklenic (2005) high-order polynom fits were used instead of spline-smoothing, thus a comparison of the outcome of the two fitting routines is also possible.
}

unknown ratio of coronal to photospheric magnetic field (Asai et al. 2004, estimated $a \sim 0.2$ ). Furthermore, note that the energy release rate $\dot{W}$ is only proportional to the real Poynting flux $S$ ( $\dot{W}=S A ; A$ is the area of the reconnection site).

In Figs. 5 and 6 , the results for paths $\mathrm{A}, \mathrm{BN}$, and $\mathrm{BS}$ and for $\mathrm{CN}, \mathrm{CS}$, and $\mathrm{D}$, respectively, are presented. The gray vertical bars act as a visual accentuation of the peaks in the full-disk HXR 20-60 keV time profile that is presented in row 7 of Figs. 5 and 6. For example, the dark-gray bar in the left column of Fig. 5, designated as A, means that the derived time profiles that are presented in this column, were obtained by tracking the northern flare ribbon across path A, which crossed the location of HXR burst $A$ in its center (cf. Fig. 2a), whereas the other bars highlight HXR bursts B, C, and D, which were not directly crossed by path A. However, since the locations of the HXR bursts were very close to one another, a particular ribbon tracking path could cross more than one HXR burst site. In these cases, however, it did not cross other burst sites at their center, where the bulk of the energy is deposited, but instead traversed their periphery; e.g., ribbon-tracking path A also crossed the edge of HXR burst $\mathrm{C}$, as well as the elongated region of HXR burst B (see Fig. 4).

In the first row of Figs. 5 and 6, the temporal evolution of the ribbon front distance $d$ from the locally defined inversion line is given, with the solid lines representing the spline fits of the profiles. Row 2 is the apparent ribbon velocity $v$ (time derivative of the spline fit to the curve $d$ shown in row 1), and row 3 displays the absolute value $B$ of the line-of-sight magnetic field strength at the ribbon front, multiplied by a scaling factor of 1.56 , which accounts for the underestimation of MDI magnetic field measurements (Berger \& Lites 2003). Here again, the solid lines are the spline fits of the measured profiles. Rows 4 and 5 show the derived electric field $E=v B$ and the Poynting flux $S=v B^{2} / \mu$, respectively. The solid $E$-profiles in row 4 are the product of the ribbon velocity $v$ of row 2 and the solid magnetic field profiles $B$ of row 3 , whereas the diamonds in row 4 are the product of the ribbon velocity and the measured $B$-values (diamonds) of row 3 . The same is valid for the Poynting flux profiles $S$ in row 5, except the fact that the square of the magnetic field strength was multiplied by the ribbon velocity. Finally, rows 6 and 7 show HXR time profiles that act as proxies for the local reconnection rate $E$ and the energy release rate $\dot{W}$ (Poynting flux $S$ ), respectively. Row 6 shows the RHESSI HXR 20-60 keV subregion time profiles. They were derived from that area, where the HXR burst that was directly crossed by the associated ribbon-tracking path, was situated, e.g., the $20-60 \mathrm{keV}$ subregion time profile in Col. 3 of Fig. 6 was constructed from the area that is marked by the dashed rectangle in Fig. 2 d. Row 7 shows the $20-60 \mathrm{keV}$ RHESSI full-disk HXR time profile.

Figures 5 and 6 show that in 5 out of 6 tracking paths, the $E$ and $S$ profiles exhibit peaks that are roughly simultaneous with the particular HXR burst. Only the peak that appeared by tracking the southern ribbon along path CS was not closely associated with HXR burst $C$, but arose delayed by about $2-3$ min. Despite this exception, we find that by tracking the flare ribbons along paths that cross the central region of a particular HXR burst site (main energy deposition site), these bursts can be reproduced in the derived local reconnection rate $E$ and the Poynting flux profile $S$, which is proportional to the energy release rate $\dot{W}$.

Moreover, other HXR bursts, which are not centrally crossed but peripherally traversed by a particular ribbon-tracking path, also appeared in the derived $E$ and $S$ profiles, most prominently peak $C$ in path $A$ and peak $A$ in path $B N$ (left and middle columns of Fig. 5, respectively), peak B in path $\mathrm{CN}$ and peak $\mathrm{C}$ 

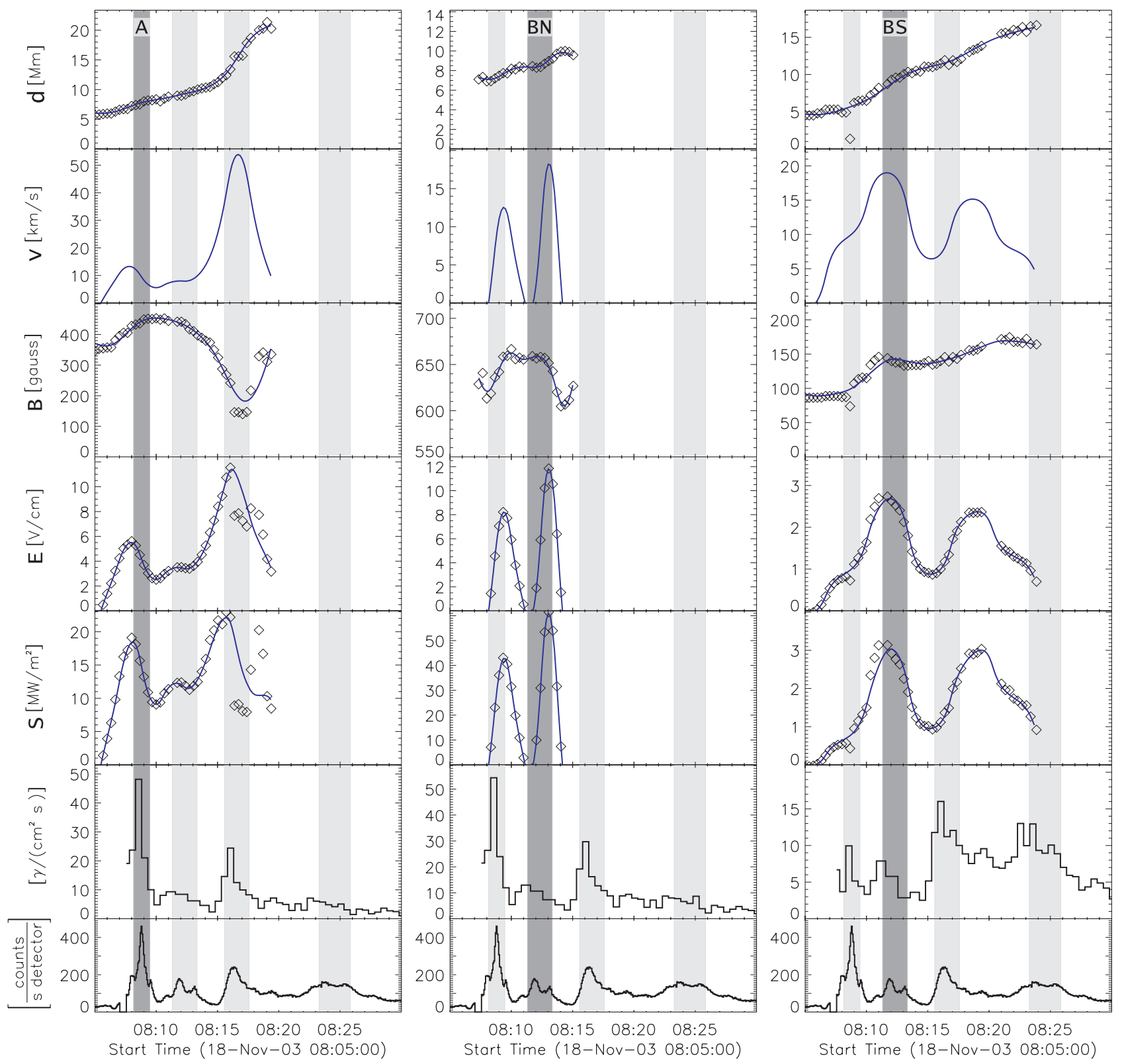

Fig. 5. Ribbon tracking results for paths A, BN, and BS. Dark-gray bars highlight the HXR burst that was directly crossed by the corresponding tracking path. Light-gray bars mark the other three HXR bursts. Row 1: temporal evolution of the ribbon-front distance from the locally defined inversion line. Solid line: spline fit. Row 2: apparent ribbon velocity. Row 3: diamonds: absolute value of the MDI photospheric line-of-sight magnetic field strength at the ribbon front scaled by 1.56 (see Berger \& Lites 2003). Solid lines: spline fit. Row 4: electric field or local reconnection rate. Solid lines: product of row 2 with the solid lines from row 3. Row 5: Poynting flux or energy release rate, respectively. Solid lines: product of row 2 with square of the solid lines from row 3. Row 6: 20-60 keV RHESSI HXR subregion light curves derived from the area where the HXR burst that was directly crossed by the associated ribbon tracking path was situated (the dashed rectangle in Fig. 2d marks such an area). Row 7: 20-60 keV RHESSI full-disk HXR time profile.

in path D, (left and right columns of Fig. 6, respectively). Again, peak $\mathrm{C}$ in the southern ribbon is an exception. By tracking the southern ribbon along path BS, a second peak arises additionally to peak B (right column of Fig. 5). This peak is not closely associated with any of the HXR bursts. It is closest in time to burst $\mathrm{C}$, but about 2-3 min delayed, similar to peak C (compare Fig. 6, middle column).

In the following, the peak values of the $E$ and $S$ profiles are given in $\mathrm{V} \mathrm{cm}^{-1}$ and $\mathrm{MW} \mathrm{m}{ }^{-2}$, respectively. They were obtained by tracking the ribbons along paths that centrally crossed a particular HXR burst site, so these are the peak values that were derived around the dark-gray bars. Local reconnection rates amount to 5.5 (peak A), $11.8(\mathrm{BN}), 2.7(\mathrm{BS}), 7.9(\mathrm{CN}), 6.9(\mathrm{CS})$, and 8.1 (D) $\mathrm{V} \mathrm{cm}^{-1}$. These values are comparable with values that were reported for M-class flares in previous studies (Wang et al. 2003; Qiu et al. 2004; Fletcher et al. 2004; Jing et al. 2005). The peak values of the estimated energy release rates amount to 18.4 (A), $61.0(\mathrm{BN}), 3.0(\mathrm{BS}), 12.6(\mathrm{CN}), 31.7(\mathrm{CS}), 13.2$ (D) $\mathrm{MW} \mathrm{m}^{-2}$. We emphasize that the real Poynting flux is only proportional to the energy release rate, and the proportionality constant is unknown.

A comparison of the $E$ and $S$ peak values with those of the HXR full-disk time profile shows that there is no clear correlation; e.g., peak A is distinctly highest in the HXR time profile but 

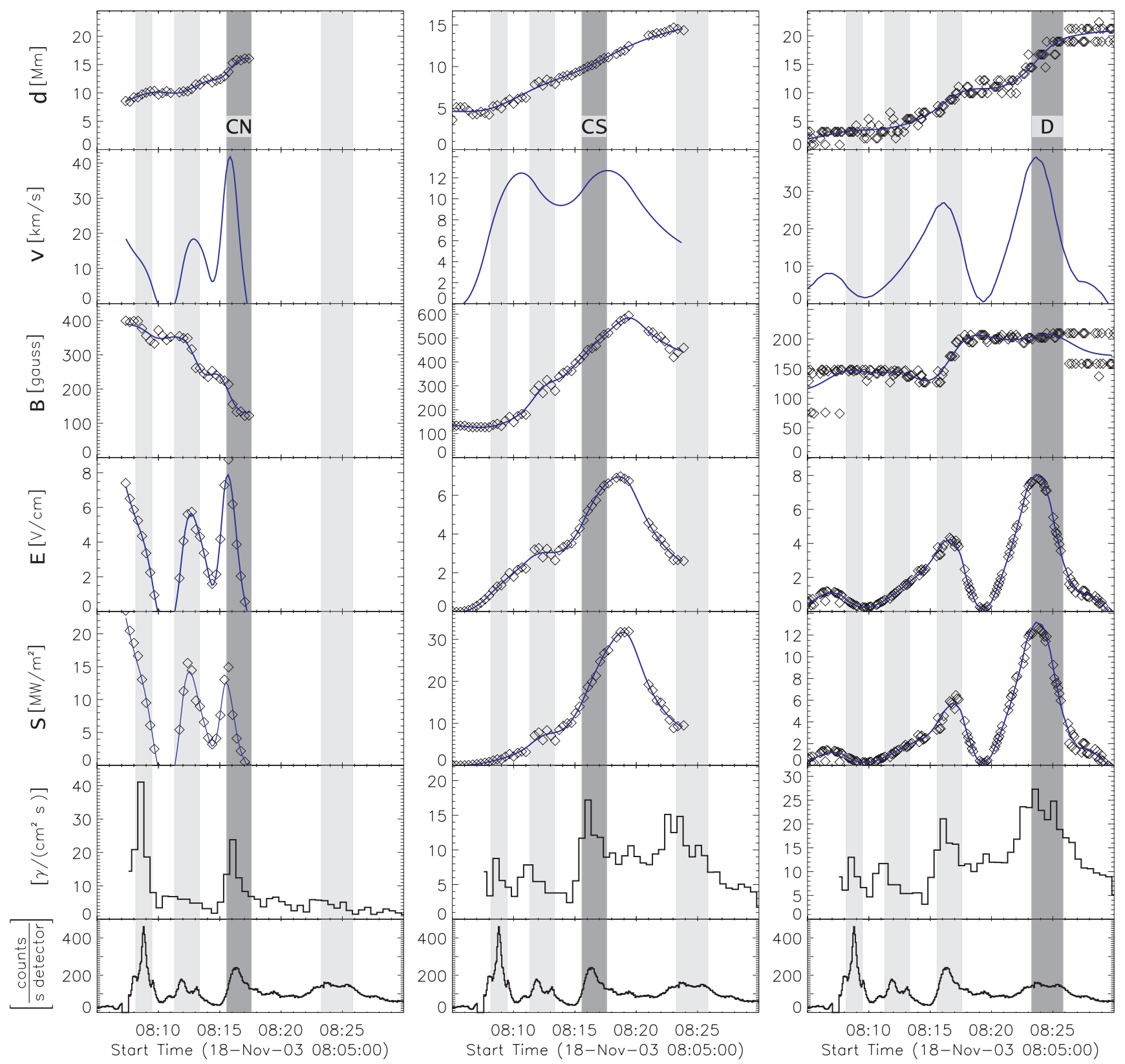

Fig. 6. Same as in Fig. 5 but for paths CN, CS, and D. Note that in path D (analyzed in $\mathrm{H} \alpha$ ) the diamonds are more scattered/noisy than in paths CN and CS (analyzed in TRACE).

not highest in $E$ and $S$, whereas peak B is lowest in HXR, but $\mathrm{BN}$ is highest in both $E$ and $S$. The correlation gets better when comparing the $E$ and $S$ peak values with those of the HXR subregion imaging light curves, e.g., peaks CS and D show up much more clearly in those HXR subregion profiles that were derived from the HXR burst CS and D areas, as they do in the full-disk profile. However, we note that also in this case the correlation is still not unique; i.e., higher HXR subregion peak values are not necessarily associated with higher peaks in $E$ or $S$.

Furthermore, Figs. 5 and 6 show that both the ribbon velocity and the magnetic field strength at the ribbon front location are essential for determining the local reconnection rate, since both quantities act together, as it is stated by Eq. (1). Asai et al. (2004) report decreasing ribbon separation speeds at the time/location of the HXR bursts. However, in all these cases the flare ribbons swept areas of increasing magnetic field strength, so the ribbons were slowed down when entering stronger magnetic field areas. In the present study, both possibilities occurred, namely, decreasing as well as increasing flare ribbon velocities at the times of the HXR bursts. The magnetic field strength at the ribbon front location seemed to be the decisive factor in this context. The ribbons speeded up while sweeping areas of decreasing magnetic field strength, and they slowed down when the magnetic fields became stronger (cf. movie 3 of the online material, where the TRACE $1600 \AA$ ribbon separation is superimposed on the MDI magnetogram). Thus, peaks in the derived local reconnection rate and energy release rate appeared either in connection with increasing ribbon velocities and decreasing magnetic field strength values (see left column of Fig. 6, during HXR burst B, as well as burst $\mathrm{C}$, time intervals) or with decreasing ribbon velocities and increasing field strengths (see left column of Fig. 5, time interval of burst A, and right column of Fig. 6, time interval of burst C). Even a third possibility occurred, namely, a maximum ribbon speed during the HXR burst 


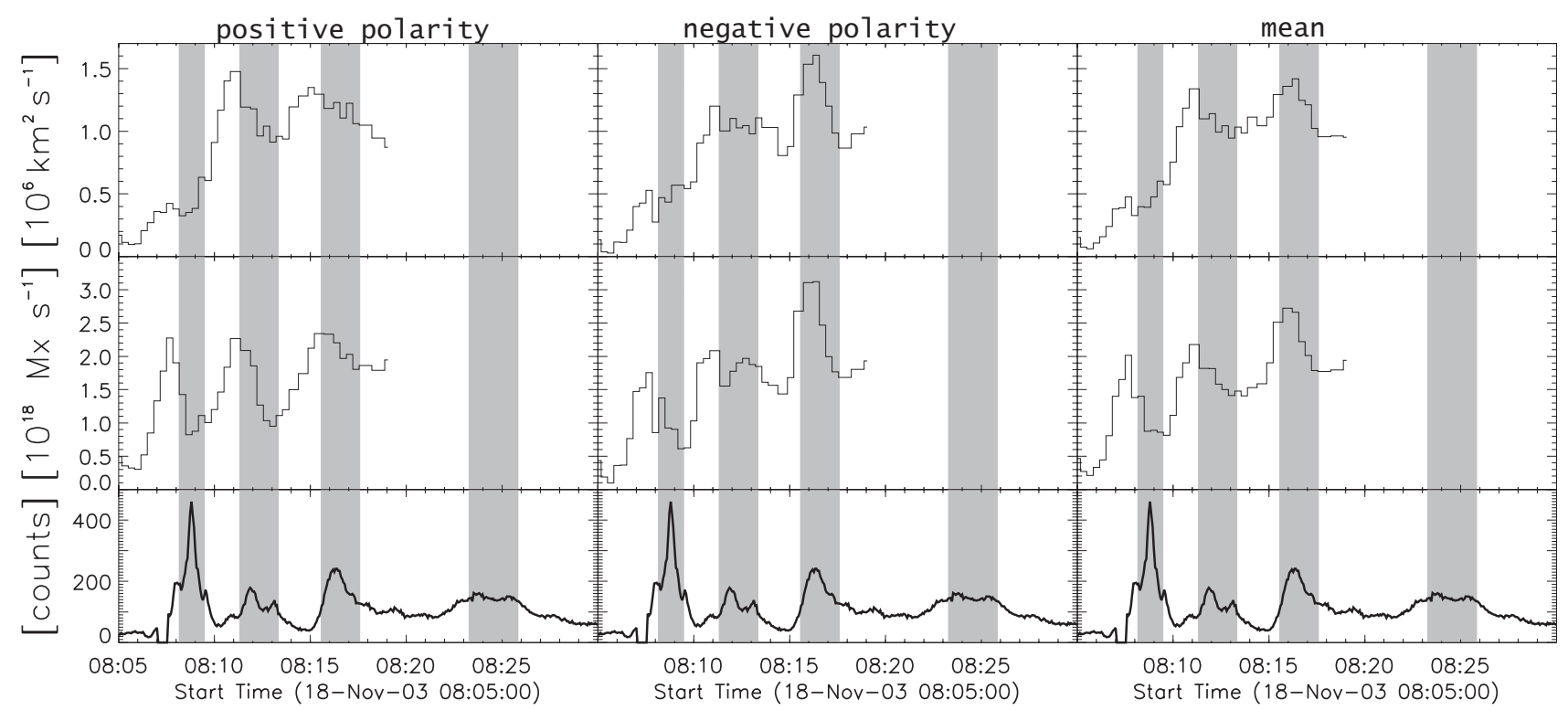

Fig. 7. Rows 1 and 2: newly brightened area and magnetic-flux change rate in the positive (left panels) and negative (middle panels) polarity domains. Right panels: mean of both polarity domains. The three peaks in the magnetic flux change rate appear around the HXR burst time intervals (gray vertical bars). Row 3: RHESSI 20-60 keV HXR full-disk time profile.

interval, while the ribbon swept an area of nearly constant magnetic field strength (see right column of Fig. 6, time interval of burst D).

\subsection{Magnetic flux change rate and flux ratio}

Rows 1 and 2 of Fig. 7 show the temporal evolution of the newly brightened area (NBA) and the magnetic flux change rate $\dot{\varphi}$ for the positive and negative magnetic polarity domains (left and middle panels), as well as the mean of both polarity domains (right panels). In order to smooth out fluctuations due to measurement uncertainties, both change rates were slightly smoothed with a 3-point boxcar average. The NBA profiles show that the amount of the newly brightened area reached its local maximum values around the time intervals of the HXR bursts (see gray vertical bars and HXR time profile in row 3 ). However, it is insufficient to consider only the NBA. The magnetic flux change rate profiles (row 2) reveal the decisive role of the magnetic field in the determination of the global reconnection rate. The three peaks that were observed in the HXR flux were reproduced much more clearly in the magnetic flux change rate than in the NBA. Note that peak A in the magnetic flux change rate of the positive polarity domain is due to the brightening in a small sunspot at the location of the two circular HXR contours in Fig. 2a. At this site there is not much NBA, since the strong magnetic field confines the ribbon movement ${ }^{2}$ (cf. movie 3 of the online material). The strong field, however, leads to a peak in the magnetic flux change rate whose level is comparable to the other two derived peaks.

However, we note that in each case the peaks in the magnetic flux change rate occurred earlier by $\sim 1 \mathrm{~min}$ than the associated HXR peaks. It is interesting to note that the amount of this time delay is comparable to the travel time of the reconnected field line from the diffusion region to the lower edge of

${ }^{2}$ For this reason, it was not possible to determine the local reconnection rate along a path that crossed this HXR burst site. the current sheet. For example, assuming a current sheet length ${ }^{3}$ of $L=50-100 \mathrm{Mm}$ and an Alfvén speed of $v_{\mathrm{A}}=1000 \mathrm{~km} \mathrm{~s}^{-1}$, one finds $t \approx L / v_{\mathrm{A}}=50-100 \mathrm{~s}$. We speculate that the delay in the HXR peaks compared to the peaks in $\dot{\varphi}$ might be explained by presuming that the sudden increase in the $\mathrm{H} \alpha / \mathrm{UV}$ emission at a given location is caused by a comparatively small number of nonthermal electrons that are accelerated immediately after the field lines that are anchored at this location enter into the diffusion region (e.g., at the slow mode shocks; Shimada et al. 1997). This results in an immediate increase in the measured NBA and magnetic flux change rate. However, the majority of nonthermal electrons, which cause the distinct increase in the observed HXR flux, might be created later, i.e., delayed for $t=L / v_{\mathrm{A}}$, and at another location, namely, at the end of the current sheet, presumably in the fast-mode standing shock at the end of the current sheet (Aurass \& Mann 2004, and references therein).

In principle, the progression of the magnetic flux change rate profiles $\dot{\varphi}$, derived from the positive and negative domains, should be identical, since at each instant equal amounts of positive and negative magnetic flux are involved in the reconnection process. However, the profiles in the left and middle panels of row 2 look different. Whereas peaks A and B were comparable to some degree in both domains, peak $\mathrm{C}$ was a good deal bigger in the negative than in the positive polarity domain $\left(2.3 \times 10^{18} \mathrm{Mx} \mathrm{s}^{-1}\right.$ vs. $\left.3.1 \times 10^{18} \mathrm{Mx} \mathrm{s}^{-1}\right)$. We note that the resulting magnetic flux change rate values, and thus also the proportion of the peak values, depend on the intensity threshold that is used to determine the NBA (the resulting NBA peak values varied by a factor of approximately 2 , and lower thresholds gave higher NBA and therefore higher reconnection rate peaks). Nevertheless, the peaks arise clearly for each threshold that is suitable to detect flare NBA. Also the time of occurrence of the derived peaks does not depend on the used threshold. The peaks that were found by means of the finally used intensity threshold

\footnotetext{
${ }^{3}$ Such a current sheet length is compatible with the observed ribbon separation of 20-30 Mm, since the angle between the slow-mode shocks bounding the reconnection outflow is very small, amounting to only a few degrees (Vršnak \& Skender 2005).
} 
value ranged from $2.0 \times 10^{18} \mathrm{Mx} \mathrm{s}^{-1}$ to $2.7 \times 10^{18} \mathrm{Mx} \mathrm{s}^{-1}$ (Fig. 7, row 2 , mean reconnection rate profile peaks). These peak values were comparable with values that were found for M-class flares by other authors (Qiu et al. 2004; Jing et al. 2005).

Finally, the ratio $R$ of converted positive vs. negative flux was determined. The magnetic flux that participated in the reconnection process within the analyzed time interval (08:00:42 UT-08:19:03 UT) was $\varphi_{+}=1.33 \times 10^{21} \mathrm{Mx}$ in the positive magnetic polarity domain, and $\varphi_{-}=-1.39 \times 10^{21} \mathrm{Mx}$ in the negative domain. The ratio $R$ added up to 1.05. In the determination of $R$, the used threshold value proved not to be a crucial factor. Deviations from the theoretically expected flux ratio never exceeded $10 \%$ with any of the tested thresholds.

Bearing the measurement errors in mind, it can be concluded that the positive and negative fluxes involved are the same. This implies that basically all reconnected field lines were rooted in the flaring region. Note that this is not necessarily always true: One can imagine a situation where some field lines that take part in the reconnection are rooted at distant locations and therefore are not identified as a part of the flare. A similar effect can occur if the energy transport is not "symmetric", i.e., if the energy is transported downward mainly along one leg of a newly reconnected field line.

\section{Summary and conclusions}

In the following we briefly summarize the main results of the analysis.

1. The peaks of the derived local reconnection rate (coronal electric field $E=v B$ ) and energy release rate (Poynting flux $S \propto v B^{2}$ ) correspond well to the peaks of the observed HXR flux (except for peak CS). Both $v$ and $B$ are essential in determining these rates, since both quantities act together. The peak values add up to $E \approx 3-12 \mathrm{~V} \mathrm{~cm}^{-1}$ and $S \approx 3-60 \mathrm{MW} \mathrm{m}^{-2}$.

2. We find a good correlation between the magnetic flux change rate $\dot{\varphi}$ (global reconnection rate) and observed HXR flux. However, we emphasize that the peaks in the magnetic flux change rate occur earlier than the associated HXR peaks by $\sim 1 \mathrm{~min}$. Based on the one event studied, it is not possible to draw conclusions on the statistical significance of this delay. However, since this delay could be related to the travel time of the reconnected field line from the diffusion region to the lower edge of the current sheet, we plan to extend this kind of study to a larger sample of flares.

3. The total magnetic flux that participates in the reconnection process is $\approx 10^{21} \mathrm{Mx}$ and is equal for positive and negative magnetic polarity domains within $5-10 \%$.

Our summary item 1 shows that the $2 \mathrm{D}$ reconnection model is applicable to the analyzed flare, since in most cases the observed HXR peaks could be related to a corresponding peak in the reconnection rate and Poynting flux. We emphasize that in more complex magnetic configurations, the velocity of the ribbon expansion is not necessarily related to the reconnection velocity. For example, in the case of reconnection taking place in strongly sheared structures, the newly brightened footpoints can show various types of "motion", generally not corresponding to an expansion away from the neutral line (e.g., Bogachev et al. 2005). Similarly, there are events in which the reconnection region expands along the neutral line (zipper-effect; Grigis \& Benz 2005), and if so, the velocity of emission patches is obviously not related to the reconnection rate at all.
We note that although we found a good time correspondence between the HXR peaks and the reconnection parameters, the amplitude of the HXR peaks itself is not directly correlated with the peak value of the reconnection rate or the Poynting flux. There are several possible reasons. First of all, the development of the flare was far from simple; i.e., it was difficult to isolate and measure fluxes of individual HXR sources. One should also bear in mind that parameter $E$ given by Eq. (1) is only a proxy for the coronal reconnection rate and that Eq. (5) only has a qualitative meaning. Moreover, the HXR flux is not simply proportional to the power in the electron beams, which strongly depends on the beam spectral index and low cut-off energy. Finally, even at the same reconnection rate and/or Poynting flux, the number of accelerated electrons is not necessarily the same, since the process of electron acceleration depends on various parameters; i.e., the proportion of thermal energy, plasma kinetic energy, and the energy of nonthermal particles can be different in different situations.

Acknowledgements. C.H.M., A.M.V., and A.H. gratefully acknowledge the Austrian Fonds zur Förderung der wissenschaftlichen Forschung (FWF grants P15344) for supporting this project.

\section{References}

Asai, A., Masuda, S., Yokoyama, T., et al. 2002, ApJ, 578, L91

Asai, A., Yokoyama, T., Shimojo, M., et al. 2004, ApJ, 611, 557 Aurass, H., \& Mann, G. 2004, ApJ, 615, 526

Berger, T. E., \& Lites, B. W. 2003, Sol. Phys., 213, 213

Bogachev, S. A., Somov, B. V., Kosugi, T., \& Sakao, T. 2005, ApJ, 630, 561

Carmichael, H. 1964, in The Physics of Solar Flares, 451

Dennis, B. R., \& Zarro, D. M. 1993, Sol. Phys., 146, 177

Fletcher, L., Pollock, J. A., \& Potts, H. E. 2004, Sol. Phys., 222, 279

Forbes, T. G., \& Lin, J. 2000, Journal of atmospheric and solar-terrestrial physics, 62, 1499

Forbes, T. G., \& Priest, E. R. 1984, in Solar Terrestrial Physics: Present and Future, ed. D. M. Butler, \& K. N. Papadopoulos, 1

Grigis, P. C., \& Benz, A. O. 2005, ApJ, 625, L143

Handy, B. N., Acton, L. W., Kankelborg, C. C., et al. 1999, Sol. Phys., 187, 229 Hirayama, T. 1974, Sol. Phys., 34, 323

Hoyng, P., Duijveman, A., Machado, M. E., et al. 1981, ApJ, 246, L155

Hudson, H. S. 1991, Sol. Phys., 133, 357

Hurford, G. J., Schmahl, E. J., Schwartz, R. A., et al. 2002, Sol. Phys., 210, 61

Isobe, H., Yokoyama, T., Shimojo, M., et al. 2002, ApJ, 566, 528

Jing, J., Qiu, J., Lin, J., et al. 2005, ApJ, 620, 1085

Kopp, R. A., \& Pneuman, G. W. 1976, Sol. Phys., 50, 85

Krucker, S., Fivian, M. D., \& Lin, R. P. 2005, Adv. Space Res., 35, 1707

Lin, J., Ko, Y.-K., Sui, L., et al. 2005, ApJ, 622, 1251

Lin, R. P., Dennis, B. R., Hurford, G. J., et al. 2002, Sol. Phys., 210, 3

Miklenic, C. 2005, Diploma Thesis, Karl-Franzens University of Graz, Austria

Narukage, N., \& Shibata, K. 2006, ApJ, 637, 1122

Neupert, W. M. 1968, ApJ, 153, L59

Otruba, W., \& Pötzi, W. 2003, Hvar Observatory Bulletin, 27, 189

Parker, E. N. 1957, J. Geophys. Res., 62, 509

Petschek, H. E. 1964, in The Physics of Solar Flares, 425

Priest, E. R., \& Forbes, T. G. 1990, Sol. Phys., 126, 319

Qiu, J., \& Yurchyshyn, V. B. 2005, ApJ, 634, L121

Qiu, J., Wang, H., Cheng, C. Z., \& Gary, D. E. 2004, ApJ, 604, 900

Sakao, T., Kosugi, T., Masuda, S., et al. 1992, PASJ, 44, L83

Scherrer, P. H., Bogart, R. S., Bush, R. I., et al. 1995, Sol. Phys., 162, 129

Shimada, N., Terasawa, T., \& Jokipii, J. R. 1997, J. Geophys. Res., 102, 22301

Sturrock, P. A. 1966, Nature, 211, 695

Sweet, P. A. 1958, in Electromagnetic Phenomena in Cosmical Physics, IAU Symp., 6, 123

Veronig, A., Vršnak, B., Dennis, B. R., et al. 2002, A\&A, 392, 699

Veronig, A. M., Karlický, M., Vršnak, B., et al. 2006, A\&A, 446, 675

Vršnak, B., \& Skender, M. 2005, Sol. Phys., 226, 97

Vršnak, B., Ruzdjak, V., Messerotti, M., \& Zlobec, P. 1987, Sol. Phys., 111, 23

Wang, H., Qiu, J., Jing, J., \& Zhang, H. 2003, ApJ, 593, 564

Yokoyama, T., Akita, K., Morimoto, T., Inoue, K., \& Newmark, J. 2001, ApJ, 546, L69 